\title{
Instrumentos para Evaluar Conocimientos, Actitudes y Prácticas en Salud Oral para Padres/Cuidadores de Niños Menores
}

\author{
Instruments for evaluating oral health knowledge, attitudes and \\ practice for parents /caregivers of small children
}

\author{
Stefania Martignon, Gloria Bautista-Mendoza, María C. González-Carrera, \\ Gloria I. Lafaurie-Villamil, Veicy Morales y Ruth Santamaría
}

\begin{abstract}
Unidad de Investigación en Caries UNICA-B, Universidad El Bosque. Bogotá, Colombia. smartignon@yahoo.com, bautistagloria@unbosque.edu.co,gonzalezmaria@unbosque.edu.co, lafauriegloria@unbosque.edu.co, veicymo@hotmail.com, ruthsantamaria@etb.net.co
\end{abstract}

Recibido 26 Enero 2007/Enviado para Modificación 28 Noviembre 2007/Aceptado 14 Marzo 2008

\section{RESUMEN}

Objetivos Construir tres instrumentos para evaluar conocimientos, actitudes y prácticas en salud oral de padres/cuidadores de niños/as de 0-5 años de estrato socioeconómico bajo; Evaluar la confiabilidad de los instrumentos por medio de su consistencia interna y análisis de ítems.

Métodos Se construyeron tres instrumentos de conocimientos, actitudes y prácticas en salud oral de padres/cuidadores de niños/as de 0-5 años, estrato socioeconómico bajo, localidad Usaquén-Bogotá, Colombia. Se ejecutó una prueba con 47 padres/cuidadores, determinando la confiabilidad de los instrumentos en términos de consistencia interna, grado de conocimientos, actitudes y prácticas de los padres/cuidadores; en una sub-muestra se realizó análisis cualitativo (verificación y comprensión del contenido). La confiabilidad fue evaluada con coeficiente Alfa-de-Cronbach. Para mejorar construcción y comprensión de preguntas se efectuó análisis de ítems utilizando cuatro criterios: índice de homogeneidad corregido (IHc), tendencia de respuesta, correlación entre ítems y análisis cualitativo.

Resultados El coeficiente Alfa-de-Cronbach para los instrumentos de conocimientos, actitudes y prácticas fue de $0,82,0,80$ y 0,62 respectivamente. La evaluación del nivel de conocimiento, actitudes y prácticas en los examinados fue aceptable (60\%, $55 \%, 91 \%$, respectivamente).

Conclusiones Este estudio encontró como confiables dos de los tres instrumentos que diseño y evaluó (conocimientos y actitudes) e hizo rediseño de los tres instrumentos. Estos son una herramienta valiosa y pueden ser utilizados en futuros estudios, tanto descriptivos como evaluativos de programas preventivos.

Palabras Clave: Conocimientos, actitudes, prácticas, confiabilidad, (fuente: DeCS, BIREME). 


\begin{abstract}
Objectives Designing three instruments for evaluating oral health knowledge, attitudes and practice in parents/caregivers of low social-economic status 0-5 yearolds. Evaluating the instruments' reliability in terms of internal consistency and analysing items.

Methods Three instruments were constructed for evaluating low social-economic status 0-5 year-olds' parents/caregivers' oral health knowledge, attitudes and practice in the municipality of Usaquén, Bogotá, Colombia. 47 parents/caregivers were given a test establishing the instrument's reliability in terms of internal consistency and the adults' level of knowledge, attitudes and practice. A sub-sample was qualitatively analysed (content verification and understanding). Reliability was evaluated using Cronbach's alpha coefficient. Items were analysed for improving constructing and understanding the questions, taking four criteria into account: corrected homogeneity index $(\mathrm{CHI})$, response trend, correlation between items and qualitative analysis.

Results Cronbach's alpha coefficient for knowledge, attitudes and practice was $0,82,0,80$ and 0,62 , respectively. Participants' level of knowledge, attitudes and practice was acceptable (60 \%, $55 \%$ and $91 \%$, respectively).

Conclusions This study found two out of the three evaluated instruments to be reliable (knowledge and attitudes); all three of them were then redesigned. The resulting instruments represent a valuable tool which can be used in future studies for describing and evaluating preventative programmes.
\end{abstract}

Key Words: Instrument, knowledge, attitude, reliability, (source: MeSH, NLM).

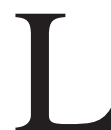

os cambios en salud oral en Latinoamérica están ligados al trabajo con y desde la comunidad (1). Las representaciones sociales de salud oral ayudan así en la planeación de programas preventivos (2).

Un instrumento válido y confiable busca ser de fácil manejo, comprensible y de práctico análisis (3-4). Su diseño debe ser compatible con los recursos disponibles (3-5). Su calidad incluye evaluación de: validez, confiabilidad amplitud de rango, capacidad responsiva, utilidad y practicidad (3-4).

En 2003 se diseñó una encuesta para evaluar conocimientos, actitudes y prácticas en salud oral de padres/cuidadores de niños de estrato socioeconómico bajo de 0-5 años, localidad Usaquén, Bogotá (6), que conjuntamente evaluaba los tres aspectos conceptualmente diferentes y no reportaba validez y confiabilidad. Así, los propósitos de esta investigación fueron, a partir de ese instrumento: 1. Construir tres instrumentos para evaluar conocimientos, actitudes y prácticas en salud oral de padres de familia/cuidadores de niños/as de 05 años de estrato socioeconómico bajo; 2. Evaluar su confiabilidad en términos de consistencia interna y análisis de ítems. 


\section{MÉTODOS}

Del instrumento preliminar (6) de 36 preguntas de conocimientos, actitudes y prácticas, se eligieron enunciados conceptualmente apropiados (7) y aprobados por su análisis (6). Los expertos definieron variables de concepto y construyeron tres instrumentos línea base de conocimientos, actitudes y prácticas, con las siguientes características:

De conocimientos: 25 preguntas dispuestas en 83 ítems; escala de medición dicotómica (8); rango de puntaje/sujeto: 0-83 puntos (ítems); clasificación de conocimiento: -escaso: 0-50, -aceptable: 5-66 y, -bueno: 67-83. -De actitudes: 17 preguntas en 29 ítems; escala de medición tipo Likert de cinco puntos (9); rango de puntaje: 0-116 puntos; clasificación de actitud: -mala: 0-58, aceptable: 59-87 y, -buena: 88-116. -De prácticas: 47 preguntas dispuestas en 49 ítems; escala de medición de Likert de cinco puntos (10); rango de puntaje posible por sujeto: 0-188 puntos; clasificación de práctica: -mala: 0-94, -aceptable: 95-141 y, -buena: 142-188.

Para determinar la comprensión y verificar el contenido de los instrumentos, se probaron en tres padres de familia de Usaquén. Se realizó evaluación cualitativa por pregunta, con grabación y transcripción para triangulación y análisis. Se evaluó la capacidad/facilidad para entender la idea de las preguntas.

Para determinar confiabilidad de los instrumentos en términos de consistencia interna, se utilizó un muestreo no probabilístico por conveniencia de 47 padres/cuidadores (previo consentimiento informado) de niños de $0-5$ años de estrato socioeconómico 1-3, localidad Usaquén. Su diligenciamiento fue orientado por seis examinadores entrenados. La consistencia interna se determinó con el coeficiente Alfa de Cronbach (4). La matriz de correlación entre ítems fue la base para su cálculo. Se consideraron adecuados valores superiores a 0.8 (3). Para el análisis de ítems, 'pregunta' se refirió a un enunciado referente a un tema; podía constar más de un ítem de respuesta. Según la matriz de correlación, se eliminaron ítems de correlación superiores a 0,85 y significativa $(\mathrm{p}<0,05)$ por redundancia (11). Para detectar ítems indiferenciadores se determinó el Indice de Homogeneidad corregido (IHc) (9) y la tendencia de respuesta en una misma dirección superiores a $95 \%$. Se consideraron adecuados índices de homogeneidad superiores a 0,2 y significativos $(\mathrm{p}<0,05)$. Para eliminar un ítem, éste debía cumplir con dos o más criterios: - no presentar verificación y comprensión de contenido, - IHc bajo y no significativo (p>0,05), - co- 
rrelación entre ítems superiores a 0,85 y significativa $(\mathrm{p}<0,05)$ y, - tendencia de respuesta igual dirección superiores a 95 \%. Se evaluó el grado de conocimientos, actitudes y prácticas de padres /cuidadores, ingresando la información en una base de datos Microsoft Excel ${ }^{\circledR}$ y categorizándola según puntuación; se analizó con el software SPSS versión 13.0®. Se determinaron frecuencias absolutas y relativas (porcentajes) del grado de conocimientos, actitudes y prácticas.

\section{RESULTADOS}

Conocimientos: El instrumento línea de base tenía 25 preguntas en 83 ítems. No hubo comprensión de contenido en las preguntas No. 6 y 17: "(Responda esta pregunta sólo si contestó negativamente (NO) la pregunta No. _ _ ).". No hubo verificación del contenido en siete ítems. El coeficiente Alfa de Cronbach de consistencia interna fue 0,82. Todas las correlaciones entre ítems fueron menores a 0,8. Los índices de homogeneidad corregidos fueron 0,090,71. Del total, 83 ítems evaluados (41\%) presentaron un valor superior a 0,2 y significativo $(\mathrm{p}<0,05)$. Seis ítems presentaron tendencia de respuesta en la misma dirección mayor a 95 \%. El instrumento definitivo fue de 25 preguntas dispuestas en 78 ítems, de cinco temas de conocimientos (Tabla 1). Su diligenciamiento tuvo una duración aproximada de 35 minutos por persona. La mayoría de padres/cuidadores (60 \%) presentó un nivel aceptable de conocimiento; $24 \%$ bueno y, $16 \%$ bajo.

Tabla 1. Temas y subtemas del instrumento de conocimientos

\begin{tabular}{lll}
\hline \multicolumn{2}{c}{ Tema } & \multicolumn{1}{c}{ Subtema } \\
\hline A & Caries dental & Causas, apariencia de lesión inicial, cómo evitarla \\
B & Cepillado dental & Función principal \\
C & Dientes temporales & Importancia y cuidado; información recibida; edad de erupción y de inicio de higiene oral \\
D & Crema dental & Importancia, edad de inicio de uso, control de cantidad \\
E & Flúor & Función, conocimiento de presencia flúor sistémico en Colombia \\
\hline
\end{tabular}

$\mathrm{A}=$ Caries dental, $\mathrm{B}=$ Cepillado dental, $\mathrm{C}=$ Dientes temporales, $\mathrm{D}=$ Crema dental, $\mathrm{E}=\mathrm{Flúor}$

Actitudes: Se generó un instrumento línea-base de 17 preguntas en 29 ítems. En comprensión de contenido, las preguntas No. 1 y 2 y, las No. 13 y 14, eran similares y poco coherentes. No hubo verificación del contenido en la No. 17. En la consistencia interna se obtuvo un Alfa de Cronbach de 0,8. Las preguntas No. 1, 2, 13 y 14 presentaron correlaciones superiores a 0,85 y significativas $(\mathrm{p}<0,05)$. Los índices de homogeneidad corregidos fueron menores a 0,86.

El 72 \% de los 21 ítems presentó un valor mayor a 0,2 y significativo $(\mathrm{p}<0,05)$. Se excluyeron tres ítems por escasa comprensión y aporte. Finalmente, se 
generó un instrumento de 14 preguntas dispuestas en 26 ítems, de seis temas (Tabla 2), con un tiempo de diligenciamiento de 15 minutos/persona. La actitud fue aceptable en $55 \%$ de padres/cuidadores y buena en $45 \%$.

Prácticas: El instrumento línea-base fue de 47 preguntas en 49 ítems. No se entendieron seis preguntas y hubo confusión las que contenían la indicación referida en el instrumento de conocimientos. La consistencia interna arrojó un valor para el coeficiente Alfa de Cronbach de 0,62. La correlación entre ítems fue en todos menor a 0,85. Los índices de homogeneidad corregidos fueron menores a 0,62. El $49 \%$ de los ítems evaluados fue mayor a 0,2 y significativo $(\mathrm{p}<0,05)$. Se generó un instrumento de 28 preguntas en 30 ítems (Tabla 3), con una duración aproximada de diligenciamiento de 30 minutos/persona. La práctica se consideró aceptable en 91,5 \% de padres/cuidadores; 6,4 \% buena y 2,1 $\%$ mala.

Tabla 2. Temas y subtemas del instrumento de actitudes

\begin{tabular}{|c|c|c|}
\hline & Tema & Subtema \\
\hline A & Prioridad de dientes sanos & Más importante que: - compartir/jugar con otros niños \\
\hline B & Persona/s responsables del niño & $\begin{array}{l}\text { De la no limpieza de los dientes, de no asistir a con - } \\
\text { sulta odontológica, de pr esentar caries, de tener } \\
\text { dolor dental, de impedir ser atendido: Padres, } \\
\text { jardineras, odontólogo }\end{array}$ \\
\hline C & Miedo/ansiedad & A la consulta \\
\hline D & Cepillado dental & $\begin{array}{l}\text { No hacerlo es incorrecto, aún si usted o el niño/a } \\
\text { está/n cansado/s }\end{array}$ \\
\hline $\mathrm{E}$ & Percepción sobre sus dientes & Le gustan / está feliz con sus dientes y su sonrisa \\
\hline $\mathrm{F}$ & Percepción del niño/a sobre sus dientes & Está feliz con sus dientes o se burla de ellos \\
\hline
\end{tabular}

Tabla 3. Temas y subtemas del instrumento de prácticas

\begin{tabular}{|c|c|c|}
\hline \multicolumn{2}{|r|}{ Tema } & \multirow{2}{*}{$\begin{array}{l}\text { Subtema } \\
\text { Asiste, por lo menos } 1 \text { vez/an̄o, ha asistido por do- } \\
\text { lor/urgencia, motivo última consulta }\end{array}$} \\
\hline A & Asistencia del adulto al odontólogo & \\
\hline B & Asistencia del niño/a al odontólogo & $\begin{array}{l}\text { Asiste, edad de primera consulta, motivo última } \\
\text { consulta }\end{array}$ \\
\hline C & Higiene oral en el niño/a & $\begin{array}{l}\text { Inicio, frecuencia, persona/s a cargo, posición del } \\
\text { niño/a, momentos del dia }\end{array}$ \\
\hline D & $\begin{array}{l}\text { Crema dental para la higiene oral del } \\
\text { niño/a }\end{array}$ & Uso, quien la dispensa, cantidad \\
\hline$E$ & $\begin{array}{l}\text { Alimentación con lactancia materna, } \\
\text { biberón, complementaria }\end{array}$ & $\begin{array}{l}\text { Durante primer año, segundo año, tercer año de } \\
\text { vida; frecuencia de consumo de carbohidratos refi- } \\
\text { nados }\end{array}$ \\
\hline$F$ & $\begin{array}{l}\text { Mecanismos para calmar la inquie- } \\
\text { tud/ansiedad del niño/a o para acostarlo }\end{array}$ & $\begin{array}{l}\text { Darle chupo, juguete favorito, cargarlo, dejarlo solo, } \\
\text { televisión }\end{array}$ \\
\hline
\end{tabular}




\section{DISCUSIÓN}

En salud oral se han utilizado instrumentos de medición de conocimientos, actitudes y prácticas para acercarse al entendimiento de la realidad cotidiana (12-13). En Colombia, un ejemplo es la encuesta de representaciones sociales del Estudio Nacional de Salud Bucal III (ENSAB-III) de 1999 (14), que incluye creencias, conocimientos y prácticas de madres de 18 a 69 años, respecto a la salud bucal de los niños. A pesar de la cobertura y pertinencia del estudio y de la revisión de registros y depuración de datos, el instrumento no reporta validez y confiabilidad; tampoco contempló algunos aspectos del entendimiento actual de caries dental y el papel del entorno del niño. Así, se descartó utilizarlo, pero algunas preguntas sirvieron de referencia (6).

La encuesta de conocimientos, actitudes y prácticas inicialmente elaborada (6) contaba con una fuerte base teórica (7); sin embargo, mostraba fallas en su construcción y aplicación, que no permitían una interpretación real de datos y su extrapolación (12).

La muestra para la prueba de los instrumentos se seleccionó por conveniencia debido al tiempo necesario para contestar los tres cuestionarios (90 minutos), pero sus características eran similares al universo.

Las debilidades presentadas de los instrumentos línea-base son parte de un proceso normal de construcción de instrumentos (3) y su identificación y adecuación permite mejorarlo en validez y confiabilidad. En este estudio la mayoría de debilidades se encontraron en el instrumento de prácticas, siendo además consistente con el análisis cualitativo de los padres/cuidadores - quienes refirieron mayor dificultad con las preguntas que evaluaban prácticas de alimentación, cuyo índice de homogeneidad mostró valores menores a 0,2 y no significativos y, cuyo coeficiente Alfa de Cronbach fue inferior al límite establecido $(0,62)$. Esto pudo haber ocurrido por el alto número de preguntas e ítems. Su reducción a 28 preguntas y la corrección en redacción, puede mejorar esta situación.

La evaluación de la confiabilidad de los instrumentos por medio del coeficiente Alfa de Cronbach generó coeficientes adecuados $(\geq 0,8)$ para los instrumentos de conocimientos y actitudes, reflejando homogeneidad de ítems. En el análisis cualitativo es importante sin embargo, analizar los resultados a la luz del escaso número de padres. 
La evaluación de las respuestas de conocimientos, actitudes y prácticas fue coincidentemente aceptable, discrepando de otros estudios donde generalmente conocimientos y actitudes corresponden a categorías buenas-excelentes y, las prácticas a malas (15). Esto puede deberse a una tendencia cultural a contestar correctamente, (1), como se refleja en el ENSAB-III (14), donde a pesar de que la mayoría conoce cómo prevenir la caries, el $60 \%$ asiste a consulta por urgencia. Se recomienda hacer verificación con indicadores duros, como índice de placa bacteriana (5).

Este estudio diseñó y re-estructuró tres instrumentos para evaluar conocimientos, actitudes y prácticas en salud oral de padres/cuidadores de niños/as menores de 5 años de estrato socio-económico bajo-medio. Se sugiere probarlos en una muestra mayor y aplicarlos como base para la realización y evaluación de estrategias preventivas en salud oral •

\section{REFERENCIAS}

1. Díaz MP. El riesgo en Salud: Entre la visión del lego y el experto. Bogotá: Unibiblos; 2002.

2. Sohn W, Ismail Al, Tellez M. Efficacy of educational interventions targeting primary care providers' practice behaviors: an overview of published systematic reviews. J Public Health Dent 2004; 64(3):164-72.

3. Polit D, Hungler B. Investigación científica en ciencias de la salud. 6a Edición. México: McGraw-Hill Interamericana; 2000.

4. Nunally JC, Bernstein IH. Teoría Psicométrica. 3a Edición. New York: McGraw-Hill; 1994.

5. Martignon S, Gonzalez MC, Santamaria RM, Jacome-Lievano S, Munoz Y, Moreno P. Oral-health workshop targeted at 0-5-yr. old deprived children's parents and caregivers: effect on knowledge and practices. J Clin Pediatr Dent 2006;31(2):104-8.

6. Martignon S, González-Carrera MC, Jácome-Liévano S, Velosa-Porras J, Santamaría-Sánchez R. Conocimientos, actitudes y prácticas en salud oral de padres y jardineras de niños Hogares Infantiles: ICBF-Usaquén-Bogotá. Revista Científica - Facultad de Odontología Universidad El Bosque 2003; 9(2):46-59.

7. Nexø Public Dental Health Service. The Nexø Method: a program for dental health care [Internet]. Disponible en: http://www.nexodent.dk . Consultado Mayo del 2002.

8. Berkman L, Kawachi I. Social Epidemiology. 1st Edition. Oxford, New York: Oxford University Press; 2000.

9. Likert, R. A technique for the measurement of attitudes. Archieves of Psichology, 1932; 140:1-50.

10. Salant P, Dillman DA. How to conduct your own survey. 1st edition. New York, Chichester: John Wiley \& Sons, Inc; 1994.

11. Ferketich S. Focus on psychometrics: Aspects of item analysis. Res Nurs Health 1991; 14:165168.

12. Stewart JE, Strack S, Graves P. Development of oral hygiene self-efficacy and outcome expectancy questionnaires. Community Dent Oral Epidemiol 1997; 25:337-342.

13. Schäfer F, Nicholson JA, Gerritsen N, Wright RL, Gillam DG, Capenhurst H. The effect of oral care feed-back devices on plaque removal and attitudes towards oral care. Int Dent Journal 2003; 53:404-408.

14. Ministerio de Salud - República de Colombia. Estudio Nacional de Salud Bucal. En Ministerio de Salud, Centro Nacional de Consultoría - República de Colombia: III Estudio Nacional de Salud Bucal - ENSAB III; Tomo VII. Bogotá: Lito Servicios ALER; 1999.

15. Arnrup K, Berggren U, Broberg AG. Usefulness of a psychometric questionnaire in exploring parental attitudes in children's dental care. Acta Odontol Scand 2001; 59:14 